$\sqrt{12}$

Caso Clínico

\title{
Estenosis aórtica y lesión severa de tronco coronario: tratamiento percutáneo exitoso.
}

\author{
Alberto Barría, Gastón Dussaillant, Juan Espinosa, Mauricio González, Paula Inostroza, Rodrigo Castillo. \\ Hospital Clínico de la Universidad de Chile. Departamento Cardiovascular, Sección Hemodinamia.
}

\section{Severe aortic valve stenosis with co-existing critical obstruction of the main left coronary artery}

Successful treatment following percutaneous angioplasty (PTCA) and percutaneous trans aortic valve implantation (TAVI).

The clinical case of an 81-year-old male with severe aortic valve stenosis and critical obstruction of the main left coronary artery is presented. Due to a very high estimated surgical risk the patient underwent
PTCA of the main left trunk followed, a week later, by trans catheter implantation of an aortic valve (TAVI). The procedure was uneventful, and the clinical condition of the patient was excellent at one year (Functional class I).

Keywords: aortic stenosis, coronary angioplasty, trans aortic valve implantation. 
Presentamos un paciente de 81 años, sexo masculino con antecedentes mórbidos de hipertensión arterial, hiperlipidemia, diabetes mellitus tipo 2 y tabaquismo suspendido. Ingresado inicialmente a otro centro con diagnóstico de insuficiencia cardíaca descompensada destacando la presencia de soplo aórtico meso-sistólico y signos de congestión pulmonar. El ecocardiograma fue compatible con estenosis aórtica (EAo) severa. Fue derivado al Hospital Clínico de la Universidad de Chile (HCUCH) para resolución quirúrgica. El electrocardiograma mostró bloqueo completo rama izquierda; el ecocardiograma una fracción de eyección (FE) 34\%, estenosis aórtica severa con área de $0.5 \mathrm{~cm} 2$ (ecuación de continuidad), e insuficiencia mitral moderada a severa. La coronariografía reveló estenosis severa de tronco coronario a distal y de arteria descendente anterior ostial. Los scores de riesgo quirúrgico 10 (STS) y 15 (Euroscore). En discusión conjunta con equipos de cardiocirugía, hemodinamia y cardiología, se consideró de alto riesgo quirúrgico y fue derivado para implante percutáneo de válvula aórtica (TAVI). La tomografía computada (TC) de aorta mostró un anillo aórtico severamente calcificado, con superficie anular $494 \mathrm{~mm} 2$, perímetro anular $81 \mathrm{~mm}$, altura coronaria derecha/ izquierda 17 y $19 \mathrm{~mm}$ respectivamente. Eje iliofemoral: tortuosidad e infiltración cálcica severa con diámetro luminal mínimo de $4.5 \mathrm{~mm}$ a derecha y $5,2 \mathrm{~mm}$ a izquierdo (Figura 1).

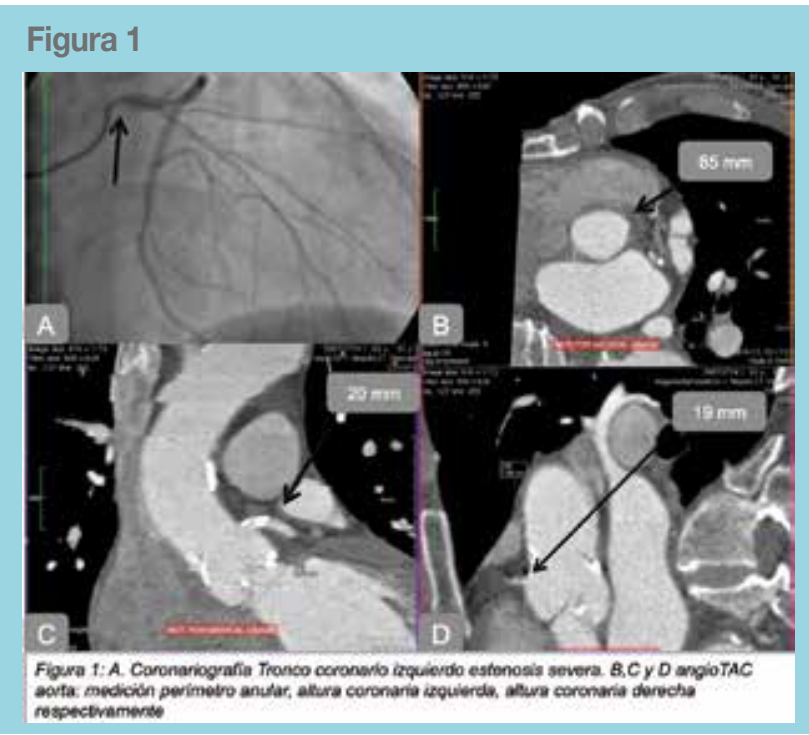

Se decidió procedimiento en dos tiempos. En un primer tiempo de efectuó angioplastía de tronco bajo soporte vital extracorpóreo (ECMO). Por acceso radial derecho se avanzó un introductor ${ }^{6}$ French (Figura 2). Se implantó

\section{Figura 2}

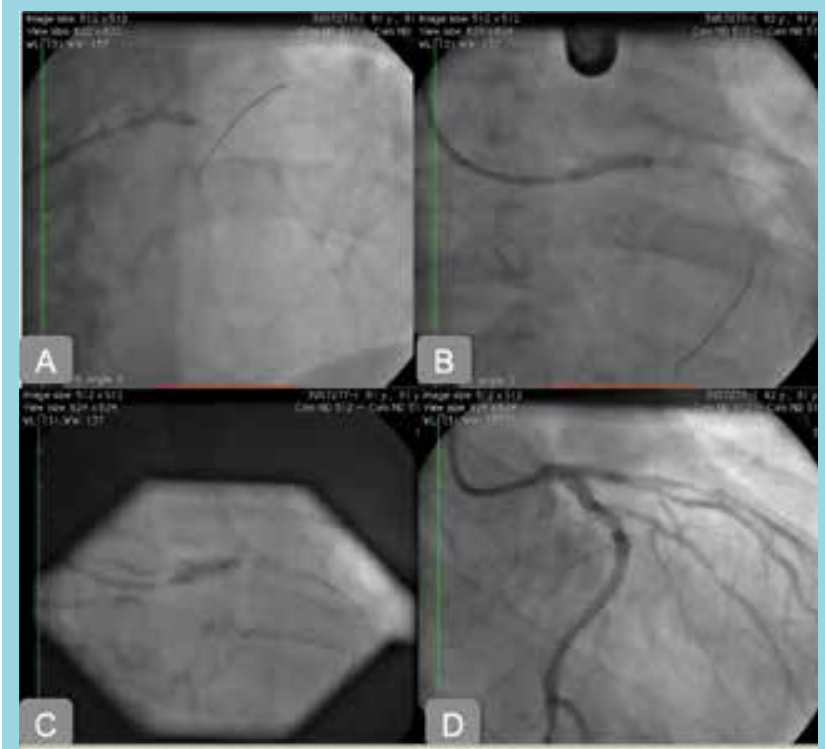

Figura 2: Angioplastia tronco coronaria. A inseroion guias arierias circunfleja y descedtente anterior, predillatación baian . 8 implante stent farmacoactivo. C Sobre-impactación stent baton NC 4, Q. D Rescitado finat tronco corcnaria.

ECMO veno-arterial con acceso venoso femoral derecho y arterial vía subclavia izquierda, para lo cual se efectuó arteriotomía e implante de prótesis de dacrón en subclavia derecha. Requirió ablación cálcica mediante dispositivo Rotablator en dos ocasiones con olivas de $1.25 \mathrm{y}$ $1.5 \mathrm{~mm}$. Se implantó stent farmacoactivo $3.5 / 15 \mathrm{~mm}$ en tronco coronario izquierdo y ADA proximal, con éxito y sin complicaciones locales ni generales, verificadas por ultrasonido intracoronario. Se retiró ECMO al finalizar de la angioplastía y el paciente evolucionó en buenas condiciones generales. Una semana post angioplastía, el paciente fue reingresado a pabellón de hemodinamia para implantación de prótesis aórtica por vía intravascular (TAVI). El procedimiento se efectuó bajo anestesia general y visión ecocardiograma transesofágico; no requirió ECMO. Se accedió por vía subclavia izquierda para implante TAVI, vía radial derecha para inserción de catéter pigtail y vía femoral derecha para sonda marcapasos transitoria. Se retrograda la válvula aórtica mediante catéter Amplatz curva 1 y guía 0,035 de punta recta. Se intercambió por guía Confida ${ }^{\circledR} 0.035$ dejando su extremo distal en ápex ventricular. Se utilizó una prótesis Evolute $\mathrm{R} 29 \mathrm{~mm} \circledast$ de acuerdo con perímetro y área anular. Se insertó la prótesis pre-montada en sistema de entrega EnveoR® por vía subclavia sin dificultades en el paso de la prótesis. Esta se desplegó bajo visión radioscópica 
y ecocardiográfica (Figura 3). Se verificó la posición y ausencia de gradiente transvalvular significativo. El paciente fue extubado en pabellón de hemodinamia y evolucionó en el post operatorio sin complicaciones locales cardiovasculares ni del acceso. El ecocardiograma post TAVI mostró FE 30\%, gradiente aórtico medio $8 \mathrm{mmHg}$, ausencia de insuficiencia aórtica, insuficiencia mitral moderada, presión sistólica pulmonar $32 \mathrm{mmHg}$. Fue dado de alta en buenas condiciones generales. Se controló clínicamente a 6 meses y estaba en capacidad funcional 2. En el control clínico a 12 meses se encontraba en capacidad funcional $1 \mathrm{y}$ sin necesidad de nuevas hospitalizaciones.

\section{Figura 3}

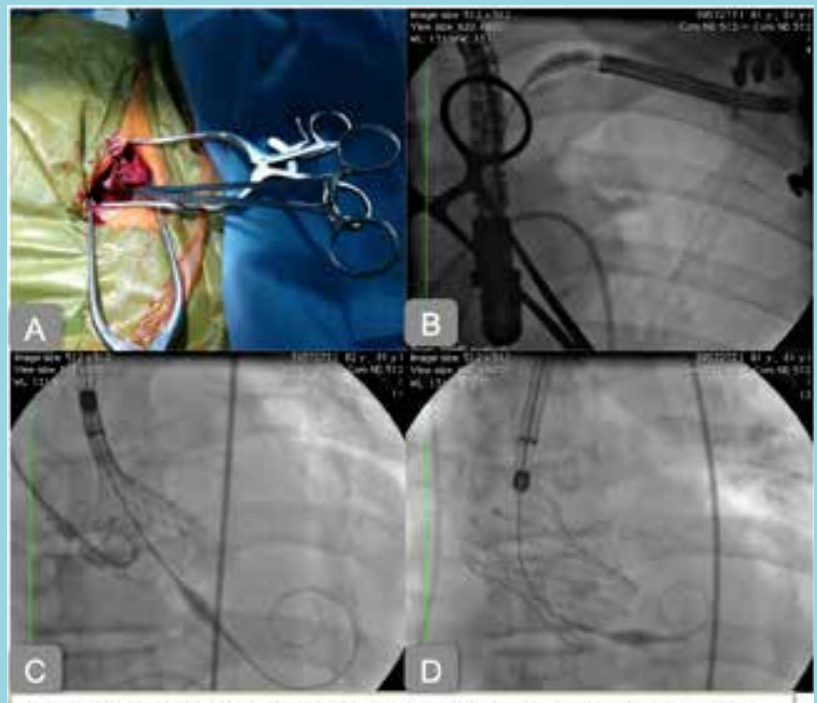

Figura 3. Procedimiento TAVI. A Acceso subclavia arteriotomie y prctesis de dacrón. B. ingresso dispositivo TAVI Evolute-R via subciavia. C. Liberacibh de protesis guada por radioscopia. D. Liboracion complote de protesis.

\section{Discusión}

En 20 a 30\% de los pacientes portadores de EAo severa no se efectúa resolución quirúrgica dado su alto riesgo operatorio o fragilidad ${ }^{1}$. En estos casos se ha implementado TAVI como un método de tratar la estenosis aórtica evitando los riesgos de la cirugía estándar. Estudios clínicos multicéntricos revelaron que TAVI es la elección en casos rechazados para cardiocirugía ${ }^{2}$ y es al menos similar en resultados a largo plazo en pacientes de riesgo moderado y alto ${ }^{3}$. En nuestro medio disponemos de escasos datos en este tipo de intervenciones, la mayor serie publicada es de 26 casos $^{4}$. En nuestro caso se adicionó la dificultad de no disponer de un acceso femoral adecuado. Es relevante que dispusimos de esta información previa a la instalación de ECMO, lo cual determinó el uso de acceso arterial subclavio. El uso de cánulas arteriales por vía femoral hubiere expuesto a complicaciones graves del ECMO como isquemia aguda de extremidades inferiores. Es igualmente relevante que, pese al alto riesgo de la angioplastía de tronco, esta se pudo llevar a cabo exitosamente vía radial con introductor 6 French permitiendo uso de ultrasonido intracoronario y Rotablator. Esto confirma que la vía radial otorga las mismas posibilidades que la vía femoral evitando el riesgo de lesión vascular iliofemoral ${ }^{5}$, que en este caso hubiese sido de pronóstico incierto $^{6}$. Se decidió efectuar el procedimiento TAVI en un segundo tiempo. En este caso la decisión fue influida por la alta complejidad del primer procedimiento. La información proporcionada por el scanner preoperatorio fue indispensable permitiendo estimar el tamaño de la prótesis a implantar, el riesgo de oclusión coronaria, el grado de calcificación central y la suficiencia del acceso femoral ${ }^{7}$. En casos con pobre acceso femoral, la vía alternativa más recurrida en los estudios iniciales correspondía a trans-apical ${ }^{8}$. En los últimos años se ha ido progresivamente disminuyendo el uso de esta vía, dada la asociación con mayor riesgo peri-procedimiento y evidencias de mayor injuria miocárdica ${ }^{9}$. En este paciente se optó por la vía subclavia, dada la mayor experiencia y comodidad del equipo quirúrgico con este acceso. Otras posibilidades actuales corresponden a trans-aórtica, axilar, carotidea y trans-caval ${ }^{10}$. Esta última se usa escasamente y su evidencia en la literatura solo corresponde a reportes de casos. Respecto a la elección del tipo de prótesis, al momento de la intervención se encontraban disponibles en nuestro medio prótesis tipo balón expansible Edwards y tipo autoexpansible Medtronic. La evidencia actual ha demostrado que ambos tipos de prótesis logran buenos resultados con mortalidad peri procedimiento menor a $2 \% 11,12$ y eventos cardiovasculares, como infarto, accidente cerebrovascular, isquemia cerebral transitoria, complicaciones vasculares del acceso y falla renal aguda menor al 5\%. La incidencia de bloqueo auriculoventricular con necesidad de marcapasos definitivo post-procedimiento es un evento adverso conocido para este tipo de procedimiento y su incidencia puede llegar hasta $25 \%$, incluso mayor en prótesis autoexpansible. En registros multicéntricos publicados recientemente, se ha visto una disminución en la tasa de implante de marcapasos posterior a la intervención TAVI ${ }^{13}$. El implante TAVI se ha efectuado predominantemente utilizando prótesis auto expansibles en Chile y en América Latina. Ejemplo de lo anterior es el uso de prótesis CoreValve ${ }^{\circledR}$ en el $80 \%$ de 
los casos del registro multicéntrico brasileño ${ }^{14}$. Es posible que en lo anterior influyan no solo consideraciones técnicas, sino también económico- administrativas.

\section{Conclusión}

El tratamiento percutáneo de un paciente con lesión severa de tronco coronario y EAo severa fue factible por vía percutánea incluyendo la realización de angioplastía de tronco por vía radial. Fue fundamental el uso del scanner de aorta para seleccionar el abordaje más seguro para el acceso del soporte ECMO y para el implante TAVI. Al momento, el paciente se mantiene en capacidad funcional 1 y sin eventos cardiovasculares a más de un año de la intervención, por tanto, podemos afirmar que su pronóstico a mediano plazo ha mejorado respecto a lo esperado en una estenosis aórtica severa.

\section{Referencias}

1. OSNABRUGGE, R.L., et al., Aortic stenosis in the elderly: disease prevalence and number of candidates for transcatheter aortic valve replacement: a meta-analysis and modeling study. J Am Coll Cardiol, 2013. 62(11): p. 1002-12.

2. LEON, M.B., et al., Transcatheter aortic-valve implantation for aortic stenosis in patients who cannot undergo surgery. N Engl J Med, 2010. 363(17): p. 1597-607.

3. SMITH, C.R., et al., Transcatheter versus surgical aortic-valve replacement in high-risk patients. N Engl J Med, 2011.364(23): p. 2187-98.

4. PINEDA F, S.B., SORE L, DECK C, TURNER E, PARRA V, BORQUEZ E, BERNAL S, SILVA J, GONZALEZ S, Uriarte P, Experiencia inicial del implante percutáneo de la válvula aórtica en el Instituto Nacional del Tórax. Rev Med Chile, 2015. 143: p. $1512-1520$

5. JOLLY, S.S., et al., Radial versus femoral access for coronary angiography or intervention and the impact on major bleeding and ischemic events: a systematic review and meta-analysis of randomized trials. Am Heart J, 2009. 157(1): p. 132-40.

6. EICHHOFER J; HORLICK E, I.J., SEIDELIN P, ROSS J, ING D, DALY P, MACKIE K, RIDLEY B, SCHWARTZ L, BAROLET A, DZAVIK V, , Decreased complication rates using the transradial compared to the transfemoral approach in percutaneous coronary intervention in the era of routine stenting and glycoprotein platelet IIb/IIIa inhibitor use: A large single-center experience. Am Heart J, 2008. 156(5): p. 864- 870.

7. TOPS, L.F., et al., Noninvasive evaluation of the aortic root with multislice computed tomography implications for trans- catheter aortic valve replacement. JACC Cardiovasc Imaging, 2008. 1(3): p. 321-30.

8. RODES-CABAU, J., et al., Long-term outcomes after transcatheter aortic valve implantation: insights on prognostic factors and valve durability from the Canadian multicenter experience. J Am Coll Cardiol, 2012. 60(19): p. 1864-75.

9. BLACKSTONE, E.H., et al., Propensity-matched comparisons of clinical outcomes after transapical or transfemoral transcatheter aortic valve replacement: a placement of aortic transcatheter valves (PARTNER)-I trial substudy. Circulation, 2015. 131(22): p. 1989-2000.

10. FROHLICH, G.M., et al., Comparative survival after transapical, direct aortic, and subclavian transcatheter aortic valve implantation (data from the UK TAVI registry). Am J Cardiol, 2015. 116(10): p. 1555-9.

11. BARBANTI, M., et al., 5-Year Outcomes After Transcatheter Aortic Valve Implantation With CoreValve Prosthesis. JACC Cardiovasc Interv, 2015. 8(8): p. 1084-91.

12. MURALIDHARAN, A., et al., Meta-Analysis of Perioperative Stroke and Mortality in Transcatheter Aortic Valve Implantation. Am J Cardiol, 2016. 118(7): p. 1031-45.

13. GILARD, M., et al., Late Outcomes of Transcatheter Aortic Valve Replacement in High-Risk Patients: The FRANCE-2 Registry. J Am Coll Cardiol, 2016. 68(15): p. 1637-1647.

14. DE BRITO, F.S., JR., et al., Outcomes and predictors of mortality after transcatheter aortic valve implantation: results of the Brazilian registry. Catheter Cardiovasc Interv, 2015. 85(5): p. E153-62. 